\title{
First-principles investigations of the magnetocrystalline anisotropy in strained fcc Co
}

\author{
G. Y. Guo* \\ Department of Physics, National Taiwan University, Taipei, Taiwan 10617, Republic of China \\ and Daresbury Laboratory, Warrington, Cheshire WA4 4AD, United Kingdom \\ D. J. Roberts and G. A. Gehring \\ Department of Physics, University of Sheffield, Sheffield S3 7RH, United Kingdom
}

(Received 1 September 1998; revised manuscript received 28 December 1998)

\begin{abstract}
First-principles calculations have been performed to evaluate the magnetocrystalline anisotropy energy as well as the phenomenologically defined anisotropy and magnetoelastic constants of strained fcc Co. We considered an applied uniaxial strain along both the [110] and [001] directions. It is found that the uniaxial strains not only induce large uniaxial and planar magnetoelastic anisotropies but also can act to suppress the cubic magnetocrystalline anisotropy. In the cubic limit, the calculated magnetoelastic coupling constant $B_{1}$ and magnetocrystalline anisotropy constant $K_{1}$ agree well with current experiments. The results are used to discuss the interesting behavior of the magnetic anisotropies as a function of film thickness observed recently in fcc $\mathrm{Co}(110) / \mathrm{Cu}$ films. In particular, it is demonstrated that in the thick Co films ( $\geqslant 50 \AA$ ) the uniaxial and in-plane anisotropies are predominantly strain induced. It is also argued that the observed abrupt transformation in the anisotropy constants at $50 \AA$ film thickness may be caused by the combined effects of the dramatic change from the isotropic in-plane strains to the anisotropic ones and the increase in the strain size as the film thickness is gradually reduced. [S0163-1829(99)07621-3]
\end{abstract}

\section{INTRODUCTION}

Ultrathin magnetic structures are commonly composed of magnetic and nonmagnetic layers that are chosen for their compatible lattice parameters, thus ensuring good epitaxy. However, small differences are unavoidable and a lattice mismatch of even a few percent can have a significant effect at this length scale. The consequences of lattice strains and the magnetoelastic anisotropy contributions that they induce have been the subject of a number of recent experimental and theoretical studies. ${ }^{1-13}$ Of particular relevance to this paper is the experimental work of Hillebrands et $a l^{8}$ and Fassbender et $a .^{9}$

A full description of the experimental details is given elsewhere ${ }^{8,9}$ but it is nevertheless useful to summarize their observations. Hillebrands et al. ${ }^{8}$ and Fassbender et al. ${ }^{9}$ considered the magnetic evolution of $\mathrm{Co} / \mathrm{Cu}(110)$ structures during their Co deposition phases. They performed Brillouin light-scattering measurements to determine the behavior of the phenomenologically defined magnetic anisotropy constants as a function of Co-layer coverage up to a thickness of $140 \AA$, which was taken to be the effective bulk limit. The high strain regime is therefore for low Co coverage and this gradually relaxes as the bulk environment is approached at higher thicknesses. Curiously, the fourth-order cubic magnetocrystalline anisotropy (commonly referred to as $K_{1}$ ) was seen to rapidly vanish for high strain, corresponding to low coverage (below about $50 \AA$ ). This breakdown is apparently accompanied by a maximum in the absolute value of the uniaxial in-plane anisotropy. Both of these effects were presumably triggered largely by the increasing strain-induced perpendicular magnetoelastic anisotropy. From this data we are led to the proposition that the presence of the uniaxial strain acts to suppress the bulk magnetocrystalline anisotropy strongly. This is surprising because according to conventional additive models each anisotropy coefficient should be independent from others - especially those of differing order and symmetry.

In a previous paper ${ }^{11}$ on the subject we were able to explain the effect in terms of a simple crystal-field model that is based upon symmetry considerations alone. Nevertheless, a first-principles approach [e.g., relativistic electronic bandstructure calculation using the spin-polarized relativistic linear muffin-tin orbital (SPR-LMTO) method ${ }^{14}$ ] to these effects is still a desirable calculation. However, the application of $k$-space band-structure methods in conjunction with a slab-supercell geometry to ultrathin systems is limited by the low symmetry that such structures by definition possess. In this paper, we side-step this difficulty, as described below, by considering only the magnetocrystalline anisotropies and magnetoelastic anisotropies due to elastic strains caused by the lattice mismatch at the interfaces. A future calculation should include both the interface and strain-induced anisotropies however the aim of this paper is to give the first quantitative account of the effects of strain. We, therefore, neglect possible interface anisotropies which are also known to contribute to the total anisotropy of an ultrathin film. Despite this limitation, we believe that our first-principles studies are significant on the following accounts. First of all, the effects of the uniaxial strains along the [110] and [001] axes on the cubic anisotropy of fcc Co have not been studied theoretically before. Also no $a b$ initio calculations for the uniaxial and planar anisotropies in the fcc Co strained uniaxially along the [110] direction have been reported to date. Second, the interesting behavior of the various anisotropy constants as a function of Co-layer coverage appears to be mainly caused by the changes in the perpendicular uniaxial strain. ${ }^{8,9}$ Therefore, the results of our calculations can, in principle, be 
directly compared with the experimental findings on thick Co films because in this case the interface contributions are generally small. Indeed, Lee et al. ${ }^{15}$ found that the consideration of magnetocrystalline anisotropy and magnetoelastic contributions alone yields a reasonably good estimate of the observed perpendicular anisotropy in $\mathrm{Co}(111) / \mathrm{Cu}(111)$ superlattices. Finally, there have been numerous first-principles calculations on bulk magnetocrystalline anisotropy energy and also on uniaxial anisotropy constant along the layer normal in multilayers, thin films and surfaces in the past decade. However, there has been little theoretical work on in-plane magnetic anisotropy in magnetic films ${ }^{16}$ other than tightbinding model calculations. ${ }^{17}$ Furthermore, there are rather few first-principles calculations on magnetoelastic anisotropies. ${ }^{7,10,12,13,16}$

\section{THEORY AND COMPUTATIONAL DETAILS}

In this paper, we consider a fcc Co film on $\mathrm{Cu}$. As mentioned above, we neglect surface and interface contributions to the anisotropy energy. This leads us to consider a strained, infinite crystal. We study the unit cell of fcc Co for the two lattice types that correspond to the $\mathrm{Co}[110]$ and $\mathrm{Co}[001]$ orientations. These are the orthorhombic and tetragonal lattices, respectively. The strain is then incorporated by varying the $c / a$ lattice parameter ratio in each case in such a way as to produce a desired percentile strain along the film normal.

The contribution to the energy which depends on the angle of magnetization for a cubic crystal perturbed by a [110] strain is given below ${ }^{8}$

$$
\Delta E=K_{1}\left(\beta_{x}^{2} \beta_{y}^{2}+\beta_{y}^{2} \beta_{z}^{2}+\beta_{x}^{2} \beta_{z}^{2}\right)+K_{\mathrm{in}} \beta_{z}^{2}-\frac{K_{\mathrm{out}}}{2}\left(\beta_{x}+\beta_{y}\right)^{2} .
$$

Here $\beta_{x}, \beta_{y}, \beta_{z}$ are the direction cosines of the magnetization relative to the crystalline axes. The [110] strain induces both $K_{\text {out }}$ and a further anisotropy in the [001]-[1 $\left.\overline{1} 0\right]$ plane and this is represented by $K_{\text {in }}$. This expression leads to the following total energies for four independent directions:

$$
\begin{gathered}
E_{[001]}=K_{\text {in }}+E_{0}, \\
E_{[1 \overline{1} 0]}=\frac{K_{1}}{4}+E_{0}, \\
E_{[110]}=\frac{K_{1}}{4}-K_{\text {out }}+E_{0}, \\
E_{[1 \overline{1} 1]}=\frac{K_{1}}{3}+\frac{K_{\text {in }}}{3}+E_{0} .
\end{gathered}
$$

These equations may be solved to obtain $K_{1}, K_{\text {in }}$, and $K_{\text {out }}$ in terms of energy differences,

$$
\begin{gathered}
K_{\mathrm{out}}=E_{[1 \overline{1} 0]}-E_{[110]}, \\
K_{\mathrm{in}}=\frac{1}{2}\left(3 E_{[1 \overline{1} 1]}+E_{[001]}-4 E_{[1 \overline{1} 0]}\right), \\
K_{1}=2\left(3 E_{[1 \overline{1} 1]}-2 E_{[1 \overline{1} 0]}-E_{[001]}\right) .
\end{gathered}
$$

Each anisotropy energy is determined by performing totalenergy calculations with the magnetization along one of these crystallographic directions and then taking combinations as specified in Eq. (3). $K_{\text {out }}$ and $K_{\text {in }}$ depend on the spin-orbit coupling to second order whereas $K_{1}$ is a fourthorder term. In the cubic limit both $K_{\text {out }}$ and $K_{\text {in }}$ vanish.

Now consider Co [001] orientation. The equivalent expression to Eq. (1) is now:

$$
\Delta E=K_{1}\left(\beta_{x}^{2} \beta_{y}^{2}+\beta_{y}^{2} \beta_{z}^{2}+\beta_{x}^{2} \beta_{z}^{2}\right)-K_{\text {out }} \beta_{z}^{2} .
$$

In this case we consider the three directions [100], [001], and [110]. The total energies for these directions are given by the following:

$$
\begin{gathered}
E_{[100]}=E_{0}, \\
E_{[001]}=-K_{\text {out }}+E_{0}, \\
E_{[110]}=\frac{K_{1}}{4}+E_{0} .
\end{gathered}
$$

The anisotropy constants are given by the following differences in this case:

$$
\begin{gathered}
K_{\text {out }}=E_{[100]}-E_{[001]}, \\
K_{1}=4\left(E_{[110]}-E_{[100]}\right) .
\end{gathered}
$$

In this case $K_{\text {out }}$ and $K_{\text {in }}$ are second and fourth order in the spin-orbit coupling, respectively, and are obtained by subtracting total energies as shown above.

For the cubic systems, the standard expression of the magnetoelastic energy is

$$
\begin{aligned}
\Delta E= & B_{1}\left(\varepsilon_{11} \beta_{x}^{2}+\varepsilon_{22} \beta_{y}^{2}+\varepsilon_{33} \beta_{z}^{2}\right) \\
& +2 B_{2}\left(\varepsilon_{12} \beta_{x} \beta_{y}+\varepsilon_{23} \beta_{y} \beta_{z}+\varepsilon_{31} \beta_{z} \beta_{x}\right) .
\end{aligned}
$$

For the [001] orientation and elastic strain, we get

$$
B_{1}=\frac{1}{3} K_{\text {out }} / \varepsilon_{11}
$$

where elastic strain element $\varepsilon_{11}=\varepsilon$ (the lateral elastic strain). For the [110] orientation, we have

$$
\begin{gathered}
B_{1}=-\frac{1}{3}\left(K_{\text {in }}+\frac{1}{2} K_{\text {out }}\right) / \varepsilon_{11}, \\
B_{2}=-\frac{1}{2} K_{\text {out }} / \varepsilon_{12},
\end{gathered}
$$

where $\varepsilon_{11}=-(1 / 2) \varepsilon$ (the lateral elastic strain) and $\varepsilon_{12}=$ $-3 \varepsilon$. Therefore, using the calculated $K_{\text {out }}$ and $K_{\text {in }}$ at each strain, we can obtain the fcc Co magnetoelastic coupling constants $B_{1}$ and $B_{2}$ as a function of the lateral strain.

We performed all-electron self-consistent electronic structure calculations for each strain considered using the spinpolarized relativistic muffin-tin orbital (SPR-LMTO) method. ${ }^{14}$ These $a b$ initio electronic structure calculations are based upon the relativistic spin-density functional theory ${ }^{18}$ with Vosko-Wilk-Nusair parameterization of the local density exchange potential. ${ }^{19}$ We obtained the magnetocrystalline anisotropy via the so-called force theorem, ${ }^{20}$ i.e., 
defined the magnetocrystalline anisotropy energy as the difference in the eigenvalue sum between the spin-polarized relativistic band structures for the different magnetization directions considered. The validity of the force theorem has been investigated theoretically and numerically by several groups $^{21-23}$ who found that the force theorem should be a good approximation to fully self-consistent total-energy calculation of anisotropy energy in both second and fourth order in the spin-orbit coupling. Because of much smaller computational effort, the force theorem has been used in most magnetocrystalline anisotropy energy calculations..$^{20,24,22,7,16,12}$

As in previous related calculations of this type $e^{24,10}$ the basis functions used were the $s, p$ and $d$ muffin-tin orbitals. The so-called combined correction terms were included in all the present calculations. The analytic tetrahedron method was used to perform the Brillouin-zone (BZ) integrations. ${ }^{25}$ The number of $k$ points over the irreducible wedge (IW) used in the self-consistent calculations was around 7000 over 2/16 BZ for the [110] orientation and 3800 over $1 / 16$ for the [001] orientation. For the adequate calculation of the magnetocrystalline anisotropy energy an extremely dense $k$ mesh in the irreducible wedge of the Brillouin zone is required. The number of $k$ points over the IW used was therefore approximately 66000 over 4/16 BZ for the [110] orientation and 53000 over $2 / 16$ of the $\mathrm{BZ}$ for the [001] orientation. We believe that all the calculated anisotropy constants presented below, except $K_{1}$ for the [110] orientation, are well converged with respect to the number of $k$ points in the irreducible wedge used. For $K_{1}$ along the [110] orientation, to obtain the same accuracy as that for the [001] orientation, more than $120000 k$ points in the irreducible wedge would be needed since in this case, the system has a lower symmetry. However, calculations with such a large number of $k$ points are currently beyond the computing resources available to us. This is the reason that the calculated $K_{1}$ for the [110] orientation [see Fig. 1(a)] oscillates with the lateral strain.

\section{RESULTS AND DISCUSSION}

\section{A. Anisotropy constants versus strain}

Using the methods outlined above we obtained the anisotropy constants of a Co film as a function of the lateral strain that are shown in Fig. 1 (for the Co [110] orientation) and Fig. 2 [for the Co [001] orientation]. Initially a variety of positive and negative, i.e., extensive and compressive strains in the (110) or (001) plane were considered in the study. However, in view of the comments of de Miguel et al. ${ }^{26}$ and other characterization studies ${ }^{8,9}$ we limited ourselves to the consideration of extensive lateral strain regimes, i.e., compressive strains along the film normal. The compressive strain perpendicular to the layer was determined by the lateral strain such that the unit-cell volume remained constant. For simplicity, strain will always be referred to the elastic lateral strain in the rest of this paper unless stated otherwise. In the strained fcc Co films, the unit-cell volume is generally different from that of bulk fcc Co. However, both previous experiments $^{27,28}$ and our estimate using the experimental elastic constants for bulk $\mathrm{Co}$ and $\mathrm{Cu}$, show that the volume changes in $\mathrm{Co}$ films on $\mathrm{Cu}$ is within $1 \%$, and therefore, we neglect their effects in this paper.

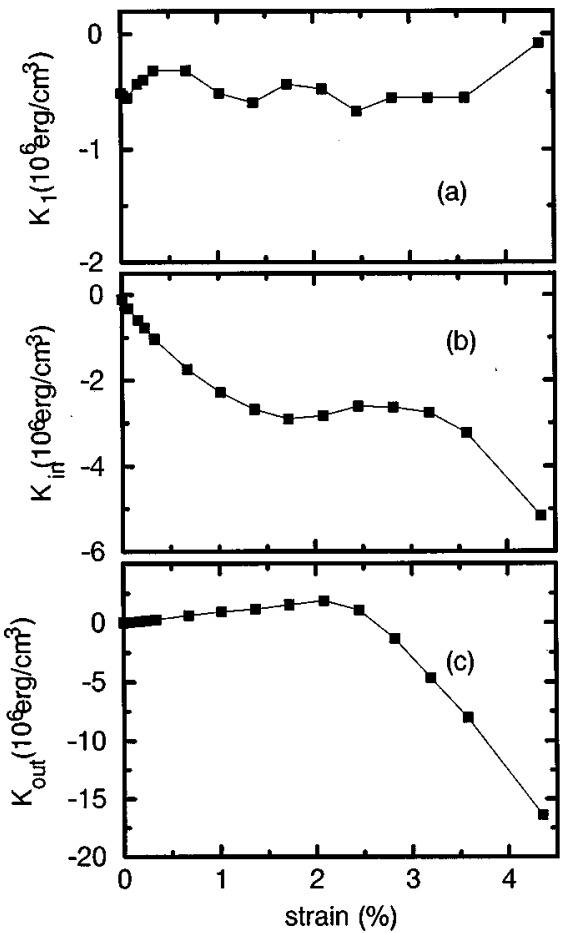

FIG. 1. Calculated cubic anisotropy constant $K_{1}$ (a), in-plane anisotropy constant $K_{\text {in }}$ (b), out-of-plane anisotropy constant $K_{\text {out }}$ (c), for a strained fcc Co as a function of the lateral strain in the (110) plane. The lines are a guide to the eye only.

Figure 1 is for the $\mathrm{Co}[110]$ orientation. For $K_{\text {out }}$ we see that initially its magnitude increases linearly as the strain increases up to $2.1 \%$. In this strain region, $K_{\text {out }}$ prefers the perpendicular magnetization (positive). In the cubic limit (zero strain) it tends to zero as it should [see Eq. 3(a)]. Interestingly, $K_{\text {out }}$ changes slope and decreases steadily as the strain further increases [see Fig. 1(c)]. $K_{\text {out }}$ changes sign at the strain of $2.6 \%$. In contrast, $K_{\text {in }}$ decreases steadily as the strain increases up to $1.7 \%$ and then remain more or less

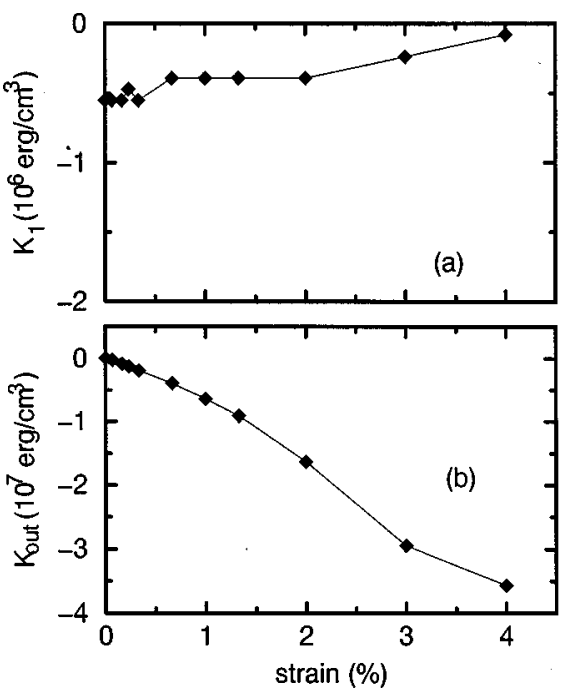

FIG. 2. Calculated cubic anisotropy constant $K_{1}$ (a), out-ofplane anisotropy constant $K_{\text {out }}$ (b), for a strained fcc Co as a function of the lateral strain in the (001) plane. The lines are a guide to the eye only. 


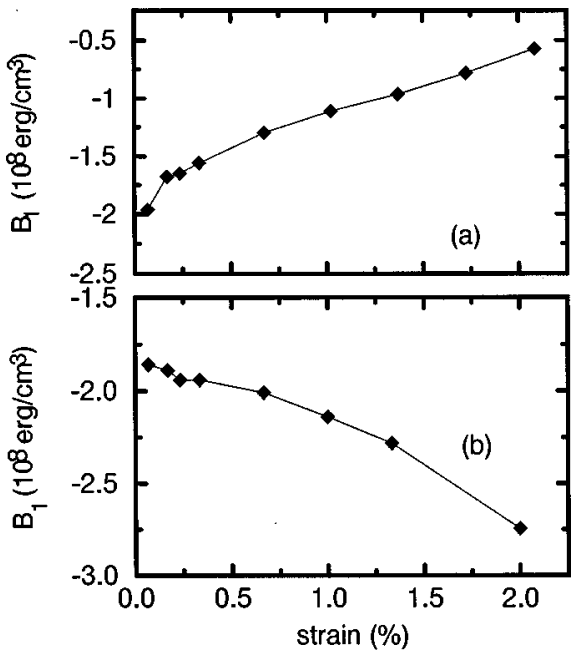

FIG. 3. Calculated magnetoelastic coupling constant $B_{1}$ for a strained fcc $\mathrm{Co}$ as a function of the lateral strain, (a) in the (001) plane, and (b) in the (110) plane. The lines are a guide to the eye only.

constant for strain up to $3.1 \%$ and then decreases steadily again as the strain further increases. However, for the Co films grown epitaxially on $\mathrm{Cu}$ substrates, the maximum strain is the mismatch between bulk fcc $\mathrm{Co}$ and $\mathrm{Cu}$ lattices, i.e., $2 \%{ }^{26}$ Therefore, for the $\mathrm{Co}$ films on $\mathrm{Cu}$ (110), we predict that $K_{\text {out }}$ and $K_{\text {in }}$ change monotonically with strain. Unlike $K_{\text {out }}, K_{\text {in }}$ does not strictly approach zero in the cubic limit [Fig. 1(b)]. The small residual value is -0.1 $\times 10^{6} \mathrm{erg} / \mathrm{cm}^{3}$ which is caused by numerical uncertainties. Indeed, increasing the number of $k$ points by 30000 reduces this value to $-0.03 \times 10^{6} \mathrm{erg} / \mathrm{cm}^{3}$ although this changes the slope of $K_{\text {in }}$ negligibly.

Using the calculated $K_{\text {out }}$ and $K_{\text {in }}$ at the smallest strain used together with Eqs. (9),(10), we obtained the fcc Co magnetoelastic coupling constants $B_{1}\left(-2.0 \times 10^{8} \mathrm{erg} / \mathrm{cm}^{3}\right)$ and $B_{2}\left(0.2 \times 10^{8} \mathrm{erg} / \mathrm{cm}^{3}\right)$. As mentioned above, in the epitaxially grown $\mathrm{Co}$ films on $\mathrm{Cu}$ substrates, the strain would range from zero to up to $2 \%$. From Fig. 1 we see that in these strain regimes, $K_{\text {out }}$ change more or less linearly. We, therefore, find no theoretical evidence for a dramatic change in the bulk magnetoelastic coupling constant $B_{2}$ in the Co films on $\mathrm{Cu}$ as has been suggested earlier. ${ }^{5}$ Indeed, the calculated $B_{2}$ are independent of the strain. Nevertheless, the calculated $B_{1}$, on the other hand, show a pronounced strain dependence [see Fig. 3(a)]. The calculated $B_{1}$ decrease monotonically in magnitude as the strain increases. At the strain of $2 \%, B_{1}$ is only $-0.6 \times 10^{8} \mathrm{erg} / \mathrm{cm}^{3}$.

Figure 1(a) shows that $K_{1}$ remains more or less constant for the strain up to $3.8 \%$. However, for higher uniaxial strain, $K_{1}$ appears to vanish, indicating its suppression due to the uniaxial perturbation in the high strain limit. This is in qualitative agreement with recent experiments ${ }^{8,9}$ and our previous simple theory. ${ }^{11,8}$ Nevertheless, the strains which can be seen in the epitaxial Co films on $\mathrm{Cu}$ (Ref. 8) are perhaps outside this very high strain region. In the zero strain limit, $K_{1}$ equals to $-0.5 \times 10^{6} \mathrm{erg} / \mathrm{cm}^{3}$ ( $-3.5 \mu \mathrm{eV} /$ atom).

The results for the tetragonal $\mathrm{Co}(001)$ system are shown in Fig. 2. The uniaxial anisotropy constant $K_{\text {out }}$ is, as might be expected from the negative value of $B_{1}$ and Eq. (8) above, is negative (i.e., preferring an in-plane magnetization) [Fig. 2(b)]. Its magnitude increases steadily with increasing strain. From the calculated $K_{\text {out }}$ we obtained the fcc Co magnetoelastic coupling constant $B_{1}$ as a function of the strain, as shown in Fig. 3(b). Interestingly, in contrast to the case of the $\mathrm{Co}[110]$ orientation [Fig. 3(a)], the calculated $B_{1}$ increases in magnitude with increasing strain. At $2 \%$ strain, $B_{1}$ is $-2.7 \times 10^{8} \mathrm{erg} / \mathrm{cm}^{3}$.

At the smallest strain used, the calculated $B_{1}$ is -1.9 $\times 10^{8} \mathrm{erg} / \mathrm{cm}^{3}$ [Fig. 1(b)]. This value is in satisfactory agreement with the value $\left(-2.0 \times 10^{8} \mathrm{erg} / \mathrm{cm}^{3}\right)$ derived above from the results at the small strain for the $\operatorname{Co}(110)$ system. Furthermore, both values compare rather well with a recent theoretical result $\left(-1.6 \times 10^{8} \mathrm{erg} / \mathrm{cm}^{3}\right)$ obtained by using the more accurate full-potential method ${ }^{7}$ and a previous experiment $\left(-1.6 \times 10^{8} \mathrm{erg} / \mathrm{cm}^{3}\right) .{ }^{29}$ However, the theoretical $B_{2}\left(0.2 \times 10^{8} \mathrm{erg} / \mathrm{cm}^{3}\right)$ obtained above appears to be one order of magnitude too small compared with the value derived from experiments $\left(2.6 \times 10^{8} \mathrm{erg} / \mathrm{cm}^{3}\right){ }^{5}$ This would suggest that our first-principles calculations greatly underestimated the size of $K_{\text {out }}$ for the $\mathrm{Co}(110)$ systems. This is perplexing because according to Eq. (9) and Fig. 1, the calculated $B_{1}$ would disagree qualitatively with experiments should this be the case. We thus speculate that part of this large discrepancy may be attributed to the fact that the experimental value was obtained by extrapolating the experimental results on fcc Co-Pd alloys. ${ }^{5}$ Unfortunately, no other calculation or experiment on the value of $B_{2}$ has been reported so far.

Interestingly, Fig. 2(a) shows that the size of the cubic anisotropy constant $K_{1}$ decreases steadily with increasing strain. This shows a transformation from a cubic to uniaxial symmetry regime, thereby implying that the uniaxial strain has acted to suppress a supposedly unrelated anisotropy. This adds weight to the contention ${ }^{8,11}$ that anisotropies might not be treated in a purely additive manner as is often assumed. Figure 2(a) approaches a suitable value of $K_{1}$ in the cubic limit (zero strain) $\left(-0.5 \times 10^{6} \mathrm{erg} / \mathrm{cm}^{3}\right)$ or $(-3.8$ $\mu \mathrm{eV} /$ atom), in good agreement with the value $(-0.5$ $\left.\times 10^{6} \mathrm{erg} / \mathrm{cm}^{3}\right)$ from Fig. 1(a), as they should be. This is very satisfactory. Both values also compare reasonably well with the recent experimental value ${ }^{8}$ of $(-0.85 \pm 0.05)$ $\times 10^{6} \mathrm{erg} / \mathrm{cm}^{3}$, given the smallness of the quantity.

To facilitate detailed comparison of the behavior of the theoretical anisotropy constants with recent experiments ${ }^{8,9}$ we must establish the link between the uniaxial strain and the Co layer thickness. It is generally believed ${ }^{30,31}$ that in epitaxial growth, ultrathin Co films initially form a coherent structure with the $\mathrm{Cu}$ substrate and hence suffer from an in-plane strain $(2 \%)$ equal to the fcc $\mathrm{Co}-\mathrm{Cu}$ lattice mismatch. When the film thickness exceeds a certain critical thickness (say, $t_{c}$ ), this strain in the Co film is relaxed gradually as the film thickness further increases due to formation of the misfit dislocations, i.e., the Co films no longer grow coherently. Chappert and Bruno ${ }^{30}$ and also den Broeder et al. ${ }^{31}$ argued that in the incoherent growth regimes, the strains are inversely proportional to layer thickness. Consequently, we also plot in Figs. 4 and 5 the behavior of the anisotropy constants as a function of

$$
t=t_{c} \varepsilon_{0} /(2 \varepsilon)
$$




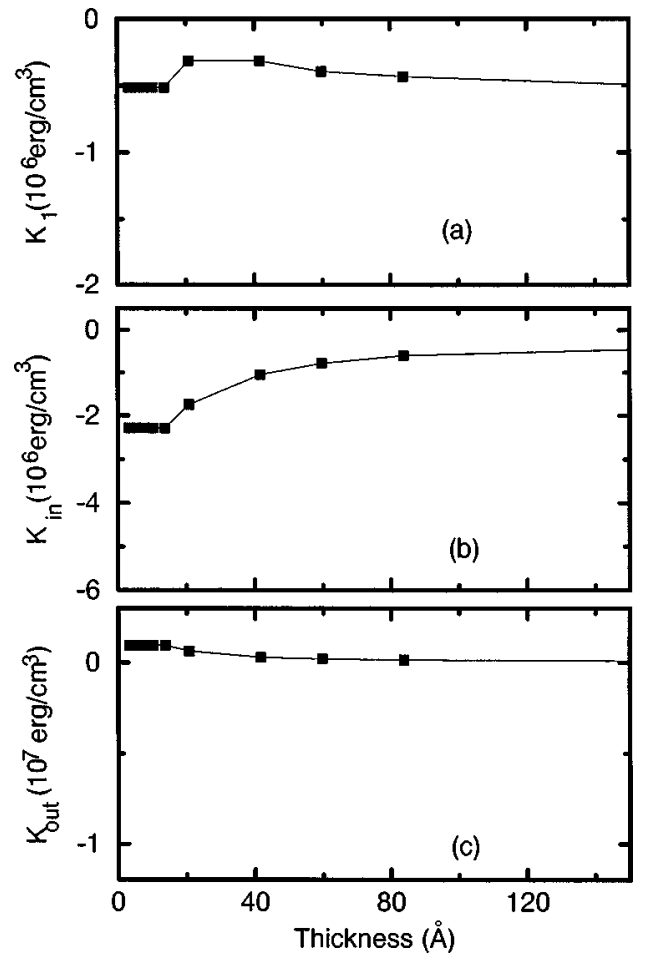

FIG. 4. Calculated cubic anisotropy constant $K_{1}$ (a), in-plane anisotropy constant $K_{\text {in }}$ (b), out-of-plane anisotropy constant $K_{\text {out }}$ (c), for a [110] oriented Co film as a function of the Co film thickness (see text). The lines are a guide to the eye only.

(1/strain $\varepsilon$ ). We assumed $\varepsilon_{0}=2 \%, t_{c}=14 \AA$ (8 ML's), and the Co film being capped by a Cu layer. ${ }^{8}$ We now compare Fig. 4 with Fig. 1 in Ref. 8. Note that the anisotropy constants should be constant below $14 \AA$ and equal to the values at $14 \AA$. First of all, the sign of the theoretical and experimental $K_{1}\left(K_{\text {in }}\right)$ agree with each other and in the thickness range $50-120 \AA$, their magnitudes are in reasonable agreement. This is rather satisfactory because of the smallness of these quantities. In other words, the magnetoelastic

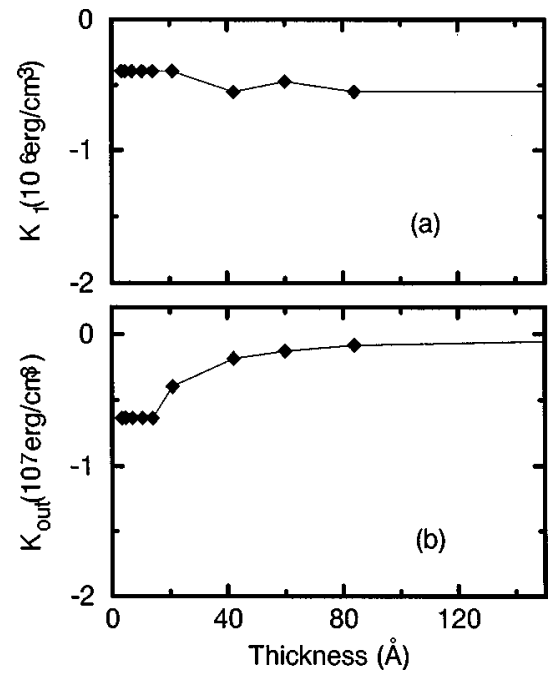

FIG. 5. Calculated cubic anisotropy constant $K_{1}$ (a), out-ofplane anisotropy constant $K_{\text {out }}$ (b), for a [001] oriented Co film as a function of the Co film thickness (see text). The lines are a guide to the eye only. anisotropies constitute dominant contributions to the total anisotropy in the fcc $\mathrm{Co}(110)$ films on $\mathrm{Cu}$ in these thickness regimes. However, the experimental $K_{\text {in }}$ and $K_{1}$ change abruptly at the Co film thickness of $50 \AA$ and then decrease dramatically as the Co film thickness further decreases. This interesting behavior is not seen in the theoretical anisotropy constants in the corresponding Co thickness range [Figs. 4(a) 4(b)]. We noticed that in the region $13-50 \AA$ the Co film grow differently from that of the film thickness greater than $50 \AA,{ }^{8,9}$ and the misfit in-plane strain appears to be anisotropic. Therefore, we are tempted to attribute the dramatic changes in the experimental $K_{\text {in }}$ and $K_{1}$ for the thinner Co films to the combined effects of both the occurrence of the anisotropic in-plane strains and the large increase in the strain size, since the anisotropy in the in-plane strain is expected to change, at least, $K_{\text {in }}$ significantly. Quantitative determination of the strains along the two orthogonal planar axes would be very helpful.

In the region $50-120 \AA$, the behavior of the experimental $K_{\text {out }}$ is qualitatively reproduced by our calculations [Fig. 4(c)]. For instance, both our calculations and experiments ${ }^{8,9}$ show that $K_{\text {out }}$ is positive. However, experimentally, as the film becomes thinner $K_{\text {out }}$ changes from positive to negative at about $50 \AA$ layer thickness. This dramatic change is not reproduced by our calculations [Fig. 4(c)]. Nevertheless, our calculations [see Fig. 1(c)] do show that $K_{\text {out }}$ changes from positive to negative at the strain of $2.6 \%$. However, the strain $(2.6 \%)$ is certainly impossible in the fcc (110) $\mathrm{Co} / \mathrm{Cu}$ films although it might be seen in, e.g., the fcc (110) Co/Au films where the misfit strain would be much larger if they could be grown. On the other hand, as mentioned before, the observed abrupt change in the anisotropy constants ${ }^{8,9}$ in the fcc Co (110) films at $50 \AA$ layer thickness is perhaps caused by the onset of the anisotropic misfit in-plane strains. The role of the observed change in the growth pattern cannot be ignored either. Further characterization measurements such as detailed strain component determination are required in order to decide whether it is purely strain-induced or otherwise. Another possible source of the discrepancy between experiments and theory could be the surface/interface contribution to the magnetic anisotropy, although the surface/interface contribution is believed to be small in this Co film thickness regime.

\section{B. In-plane anisotropy energy profile}

As mentioned earlier, Fassbender et al. ${ }^{9}$ have conducted Brillouin light-scattering experiments upon ultrathin epitaxial Co(110) films. From their analysis of spin-wave frequencies they are able to infer the angular dependence of the magnetization as a function of the angle of an in-plane applied field relative to the planar [001] direction, and therefore to directly determine the planar contribution to the anisotropy energy which they then plot for a variety of Co coverages. Their results show a transformation from planar twofold symmetry towards pseudo-four-fold symmetry as the bulk limit is approached. The results imply the suppression of the cubic anisotropy in the low-coverage high-strain regime. Accompanying this symmetry change is the switch of the easy magnetization direction from [001] to [111]. Their data was analyzed using the parametrization scheme given in 


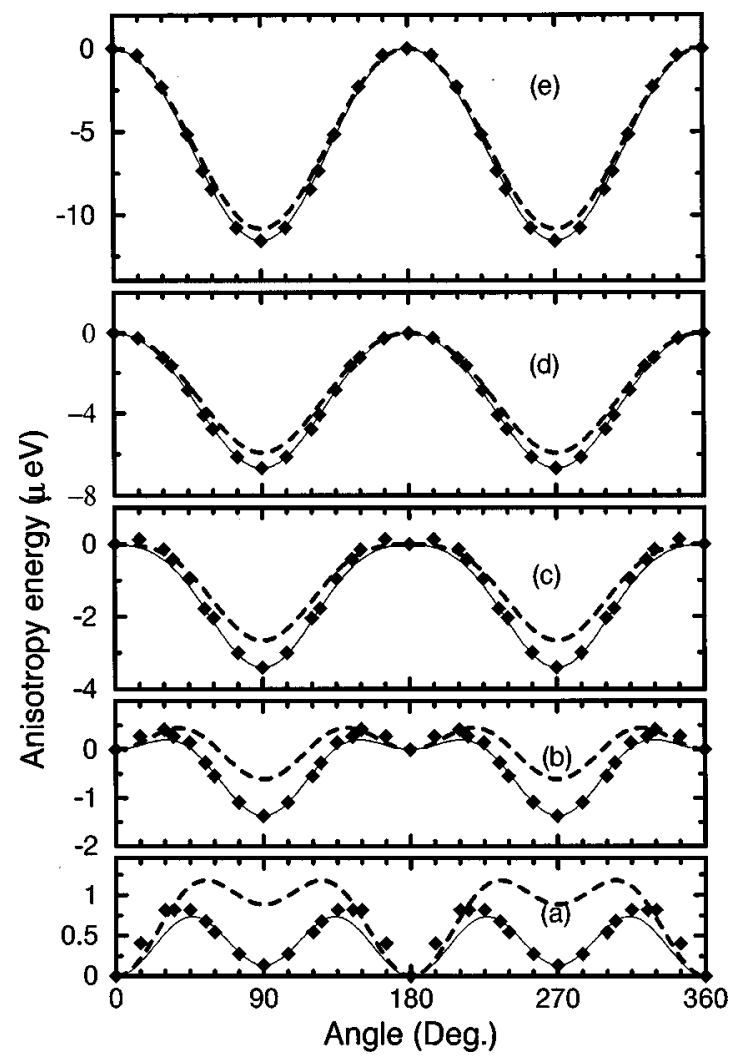

FIG. 6. Theoretical in-plane anisotropy energy $\Delta E$ of a strained fcc $\mathrm{Co}$ as a function of magnetization angle $\varphi$ (relative to the [001] direction) in the (110) plane. The maxima indicate the easy directions. From bottom to top the plots represent strains of $0,0.07,0.17$, 0.23 and $0.34 \%$, respectively. The solid curves are obtained from Eq. (12) by using the calculated $K_{1}$ and $K_{\text {in }}$, and the dashed curves by using the calculated $K_{1}$ and corrected $K_{\text {in }}$ (see text).

Eq. (1) which they found fitted the data very well. We wished to ascertain if the first-principles calculation gave an angular dependence for the anisotropy which was equally well fitted by the same scheme. In order to do this we have performed calculations that are analogous to theirs by rotating the angle of magnetization in the plane of the film and calculating the energy change. Figure 6 now gives our equivalent results from first principles calculations for a strain range $0,0.07,0.17,0.23$ to $0.34 \%$ [or layer thickness 210, 84, 60, and $42 \AA$, according to Eq. (11)]. These plots have been calculated by performing a self-consistent calculation once for each strain and then varying the angle $\varphi$ of the magnetization in steps of $15^{\circ}$. For each step an energy (eigenvalue sum) calculation for that particular magnetization direction is then performed. In Fig. 6 we plot $\Delta E(\varphi)$ $=E_{001}(\varphi=0)-E(\varphi) \quad$ (as filled diamonds) so that the maxima correspond to the easy magnetization directions. We can also get the in-plane anisotropy energy profile $\Delta E(\varphi)$ (plotted as solid curves in Fig. 6) by using the calculated anisotropy constants $K_{1}$ and $K_{\text {in }}$. From Eq. (1) we have

$$
\Delta E(\varphi)=K_{\mathrm{in}}\left(1-\cos ^{2} \varphi\right)-\frac{1}{4} K_{1} \sin ^{2} \varphi\left(1+3 \cos ^{2} \varphi\right) .
$$

There is a rather good agreement between the two anisotropy profiles for each strain shown in Fig. 6.
Figure 6 should now be compared with Fig. 6 in Ref. 9. Qualitatively, the agreement between experiment and theory is excellent. For example, like the experiments, the theoretical anisotropy profile shows diminution of the cubic pseudofourfold symmetry as the strain increases (or the Co film thickness decreases). Eventually, the anisotropy profile has the twofold symmetry (see Fig. 5). This clearly illustrates the transformation of the cubic anisotropy into a planar uniaxial anisotropy simply by virtue of a perpendicular strain upon the cubic system. Furthermore, the transformation of the easy axis to the [001] direction occurs at low strain $(\varepsilon$ $=0.2 \%$ ), i.e., at Co film thickness of about $70 \AA$ which compares rather well with the experimental transition thickness of $50 \AA .9$

However, a closer inspection reveals several discrepancies between the experiments ${ }^{9}$ and the present calculations (Fig. 6). Firstly, in the cubic limit (zero strain), the theoretical easy axis is $[1 \overline{1} 2]\left(\varphi=35.3^{\circ}\right)$ rather than $[1 \overline{1} 1](\varphi$ $=54.7^{\circ}$ ) [see Fig. 6(a)]. This discrepancy can be attributed to the numerical uncertainty because of the tiny energy difference. Indeed, the almost fourfold symmetry of the calculated anisotropy profile in the cubic limit in Fig. 6(a) is caused by the presence of the small residual $K_{\text {in }}$ due to these numerical uncertainties mentioned above. The dashed curves in Fig. 6 represent the anisotropy profiles obtained from Eq. (12) by subtracting the $K_{\text {in }}$ with this residual value. One can see now that the corrected profile (dashed line) in the zero strain correctly predicts the easy magnetization to $[1 \overline{1} 1](\varphi$ $=54.7^{\circ}$ ) [see Fig. 5(a)]. Interestingly, this indicates that the pseudo-four-fold symmetry observed at the Co film thickness of $100 \AA$ (Ref. 9) is due to the presence of both the cubic anisotropy and the small negative in-plane anisotropy $K_{\text {in }}$. Secondly, Fassbender et $a l^{9}{ }^{9}$ attribute the transformation from the pseudo-four-fold symmetry to the twofold symmetry to the drastic reduction in the cubic anisotropy $K_{1}$ as the Co film thickness getting thinner. However, we demonstrate here that if the in-plane anisotropy $K_{\text {in }}$ is greatly increased this transformation is also possible without reducing $K_{1}$.

\section{CONCLUSIONS}

We have determined the phenomenologically defined magnetic anisotropy and magnetoelastic coupling constants of fcc $\mathrm{Co}$ as a function of the uniaxial compressive strain along the [110] and [001] orientations from first-principles by using the SPR-LMTO method. These first-principles calculations gave in the cubic limit the bulk Co magnetoelastic coupling constant $B_{1}$ that agrees well with experiments $\left(-1.6 \times 10^{8} \mathrm{erg} / \mathrm{cm}^{3}\right)$. Furthermore, they produced an estimate of the bulk anisotropy constant $K_{1}=-5$ $\times 10^{6} \mathrm{erg} / \mathrm{cm}^{3}(-3.5 \mu \mathrm{eV} /$ atom $)$ which is in good agreement with current experimental determinations. ${ }^{8,9} \mathrm{We}$, therefore, conclude that the theoretical anisotropy constants presented here can be used to evaluate the magnetoelastic anisotropy contributions to the total anisotropy in fcc (110) and (001) Co films and also the effects of strains on the bulk Co magnetocrystalline anisotropy.

We have applied the results of the first-principles calculations to discuss the interesting behavior of the magnetic anisotropies as a function of film thickness in fcc (110) Co films on $\mathrm{Cu}$ observed by Hillebrands et al. ${ }^{8}$ and Fassbender 
et al. ${ }^{9}$ The first-principles calculations showed that in the thick Co films (50-140 $\AA)$ the uniaxial magnetic anisotropy perpendicular to the film and the in-plane anisotropy are predominantly strain-induced since the calculated and experimental anisotropy constants in these regimes are in satisfactory agreement. Our calculations corroborated the notion ${ }^{8,9}$ that uniaxial perpendicular strains can cause the transformation from planar twofold symmetry about the film normal to pseudo-four-fold symmetry as the bulk limit is reached. They also demonstrated that large uniaxial strains can act to suppress the supposedly unrelated cubic magnetocrystalline anisotropy [see $K_{1}$ in Figs. 1(a) and 2(a)] as well as to induce related uniaxial and in-plane magnetoelastic anisotropies, as discovered experimentally earlier. ${ }^{8,9}$ Nevertheless, the results of the present first-principles calculations showed that for the misfit isotropic in-plane strains possibly present in the fcc $\mathrm{Co}(110)$ films on $\mathrm{Cu}$, both $K_{\text {out }}$ and $K_{\text {in }}$ change monotonically as the strain increases while $K_{1}$ remain more or less constant, thereby suggesting that the observed abrupt transformation at around $50 \AA$ layer thickness in fcc $\operatorname{Co}(110)$ films on $\mathrm{Cu}$, may be due to the observed drastic change in the growth pattern (or the onset of large anisotropic in-plane strains) rather than the gradual increase in the isotropic inplane strain alone. Further experiments to determine the strain components as a function of film thickness would be very useful to clarify this interesting issue.

\section{ACKNOWLEDGMENTS}

The authors thank J. Fassbender and B. Hillebrands for stimulating discussions and critical comments on the manuscript as well as communicating their experimental results ${ }^{9}$ prior to publication. G.Y.G. acknowledges the support from the National Science Council of ROC (NSC 87-2811-M-0020035) for his sabbatical leave from Daresbury Laboratory at National Taiwan University, and he also thanks the Physics Department, National Taiwan University, especially Professor C.-R. Chang and Professor C.-D. Hu for their hospitality during his visit. D.J.R. wishes to thank the EPSRC for financial support during the period in which the work described here was performed.
*Electronic address: gyguo@phys.ntu.edu.tw

${ }^{1}$ P. Krams, F. Lauks, R.L. Stamps, B. Hillebrands, and G. Guntherodt, Phys. Rev. Lett. 69, 3674 (1992).

${ }^{2}$ B. Hillebrands and J.R. Dutcher, Phys. Rev. B 47, 6126 (1993).

${ }^{3}$ F.O. Schumann, M.E. Buckley, and J.A.C. Bland, J. Appl. Phys. 76, 6093 (1994)

${ }^{4}$ P. Krams, B. Hillebrands, G. Guntherodt, and H.P. Oepen, Phys. Rev. B 49, 3633 (1994).

${ }^{5}$ G. Bochi, O. Song, and R.C. O’Handley, Phys. Rev. B 50, 2043 (1994).

${ }^{6}$ W. Weber, C.H. Back, A. Bischof, D. Pescia, and R. Allenspach, Nature (London) 374, 788 (1995).

${ }^{7}$ R. Wu and A.J. Freeman, J. Appl. Phys. 79, 6209 (1996).

${ }^{8}$ B. Hillebrands, J. Fassbender, R. Jungblut, G. Guntherodt, D.J. Roberts, and G.A. Gehring, Phys. Rev. B 53, R10 548 (1996).

${ }^{9}$ J. Fassbender, G. Guntherodt, C.H. Mathieu, B. Hillebrands, R. Jungblut, J. Kohlhepp, M.T. Johnson, D.J. Roberts, and G.A. Gehring, Phys. Rev. B 57, 5870 (1998).

${ }^{10}$ G.Y. Guo, J. Magn. Magn. Mater. 176, 97 (1998).

${ }^{11}$ D.J. Roberts and G.A. Gehring, J. Magn. Magn. Mater. 156, 293 (1996).

${ }^{12}$ A.B. Shick, D.L. Novikov, and A.J. Freeman, Phys. Rev. B 56, R14 259 (1997).

${ }^{13}$ O. Hjortstam, K. Baberschke, J.M. Wills, B. Johansson, and O. Eriksson, Phys. Rev. B 56, 15025 (1997).

${ }^{14}$ H. Ebert, Phys. Rev. B 38, 9390 (1988).

${ }^{15}$ C.H. Lee, H. He, F.J. Lamelas, W. Vavram, C. Uher, and R. Clarke, Phys. Rev. B 42, 1066 (1990).

${ }^{16}$ M. Kim, L. Zhong, and A.J. Freeman, Phys. Rev. B 57, 5271 (1998).
${ }^{17}$ P. Bruno, Phys. Rev. B 39, 865 (1989); M. Cinal, D.M. Edwards, and J. Mathon, J. Magn. Magn. Mater. 140-144, 681 (1995); J. Dorantes-Davila and G.M. Pastor, Phys. Rev. Lett. 77, 4450 (1997).

${ }^{18}$ A.H. MacDonald and S.H. Vosko, J. Phys. C 17, 3355 (1979)

${ }^{19}$ S.H. Vosko, L. Wilk, and M. Nusair Can. J. Phys. 58, 1200 (1980).

${ }^{20}$ G.H.O. Daalderop, P.J. Kelly, and M.F.H. Schuurmans, Phys. Rev. B 41, 11919 (1990); 42, 7270 (1990).

${ }^{21}$ G.Y. Guo, W.M. Temmerman, and H. Ebert, Physica B 172, 61 (1991).

${ }^{22}$ R.H. Victora and J.M. MacLaren, Phys. Rev. B 47, 11583 (1993).

${ }^{23}$ X. Wang, D.-S. Wang, R. Wu, and A.J. Freeman, J. Magn. Magn. Mater. 159, 337 (1996).

${ }^{24}$ G.Y. Guo, W.M. Temmerman, and H. Ebert, J. Magn. Magn. Mater. 104-107, 1772 (1992).

${ }^{25}$ W.M. Temmerman, P.A. Sterne, G.Y. Guo, and Z. Szotek, Mol. Simul. 4, 153 (1989).

${ }^{26}$ J.J de Miguel, A. Cebollada, J.M. Gallego, R. Miranda, C.M. Schneider, P. Schuster, and J. Kirschner, J. Magn. Magn. Mater. 93, 1 (1991).

${ }^{27}$ H. Li and B.P. Tonner, Surf. Sci. 237, 141 (1990).

${ }^{28}$ E. Navas, P. Schuster, C.M. Schneider, J. Kirschner, A. Cebollada, C. Ocal, R. Miranda, J. Cerda, and P. de Andres, J. Magn. Magn. Mater. 121, 65 (1993).

${ }^{29}$ R.C. O'Handley (unpublished), cited in Ref. 7.

${ }^{30}$ C. Chappert and P. Bruno, J. Appl. Phys. 64, 5736 (1988)

${ }^{31}$ F. den Broeder, W. Hoving, and P.J.H. Bloeman, J. Magn. Magn. Mater. 93, 562 (1991). 